\title{
Le rôle de l'élevage dans la lutte contre la pauvreté
}

\author{
B. Faye ${ }^{1}$
}

Mots-clés

Elevage - Pauvreté - Economie Production animale - Productivité Sécurité alimentaire.

\begin{abstract}
Résumé
La lutte contre la pauvreté fait partie des priorités de nombreuses instances internationales et la place de l'élevage dans cette perspective mérite d'être précisée. La pauvreté relève de critères économiques, mais aussi sociaux et culturels, et se manifeste à plusieurs échelles (pays, régions, catégories sociales, individus). La pauvreté urbaine et la pauvreté rurale sont étroitement liées. On peut classer les éleveurs pauvres en trois catégories : les éleveurs ayant perdu leur troupeau, les éleveurs disposant d'un troupeau trop petit ou faiblement productif pour survivre, les éleveurs endettés. Ces trois catégories peuvent correspondre à différentes phases d'une paupérisation croissante. L'élevage intervient à cinq niveaux pour répondre au défi de la lutte contre la pauvreté : sécurisation, capitalisation, diversification, intégration économique, intégration sociale. Ce positionnement de l'élevage renvoie à des questions de recherche et de développement qui méritent d'être précisées.
\end{abstract}

Considérer que l'activité d'élevage peut jouer un rôle certain pour prévenir ou guérir la pauvreté c'est déjà considérer a priori que le fait d'élever des animaux est une assurance contre la misère. Cependant, les relations entre élevage et pauvreté ne peuvent s'envisager seulement sous l'angle d'une évidence positive (l'élevage permet de lutter contre la pauvreté) ou négative (l'activité d'élevage est un facteur d'appauvrissement). Comme tout moyen de production, le troupeau est soumis à des règles économiques qui échappent en partie à l'éleveur ce qui ne lui permet pas toujours d'en réguler les conséquences positives ou négatives. Aussi, on ne gagne rien à propulser l'idée d'une activité économique (l'élevage) comme remède miracle à la pauvreté, les déterminants de celle-ci dépassant largement les moyens de production que les hommes choisissent pour assurer leur existence. Toute réflexion sur le rôle de l'élevage dans la lutte contre la pauvreté ne doit pas oublier ce fait fondamental.

1. Cirad-emvt, TA30/A, Campus international de Baillarguet, 34398 Montpellier Cedex 5, France

Tél : +33 (0)4 67593703 ; fax : +33(0)4 67593825

E-mail : faye@cirad.fr
QU'EST-CE QUE LA PAUVRETE ?

Il n'est pas question dans le cadre de cet article de s'appesantir sur une définition de toute façon complexe et évolutive. La Banque mondiale s'appuie sur le seul critère de revenu - dans son rapport de 1990 sur le développement dans le monde (21), la Banque mondiale considérait comme pauvre tout individu vivant avec moins de 370 dollars par an, soit à l'époque le tiers de l'humanité -, mais ce critère est loin d'épuiser toutes les facettes de la pauvreté (5) qui relève aussi de l'éducation (analphabétisme, déscolarisation) et de la société (absence de reconnaissance sociale : minorités opprimées, castes considérées comme inférieures notamment). De fait, les institutions internationales comme la Banque mondiale considèrent la pauvreté plutôt comme une « maladie » du corps social, éthiquement « intolérable » (21), plutôt que comme une conséquence de politiques économiques engendrant un ensemble de comportements sociaux et culturels politiquement «incorrects » (5). Lewis (16) définit la pauvreté par un ensemble de caractéristiques économiques, psychologiques et sociales.

Cependant, on ne saurait réduire l'approche de la pauvreté aux seuls critères économiques (6). Ces critères considèrent, en effet, que les besoins des individus sont « donnés » et ne permettent donc pas d'étudier la manière dont se construisent les besoins, ni de discuter de la pertinence du concept de besoin. Or, ces besoins 
sont souvent difficiles à évaluer, notamment dans le domaine qui nous préoccupe. D'autant plus qu'ils varient d'une société à l'autre. Le seuil de pauvreté par exemple est estimé à partir du pourcentage du revenu médian d'une population. Ce seuil n'a donc aucune portée universelle. Les diverses facettes de la pauvreté ont donc conduit le Programme des Nations-Unies pour le développement (Pnud) à proposer une hiérarchie basée sur un indicateur combinant le revenu, l'accès à l'éducation et l'espérance de vie (elle-même étant révélatrice de l'accès aux soins), appelé indicateur du développement humain (Idh) plus proche du vécu des populations (22). Depuis 1997, le Pnud propose même un indice de pauvreté humaine (Iph) qui tient compte de l'espérance de vie, du niveau d'éducation et, c'est la nouveauté, des conditions de vie mesurées à partir de trois critères : l'accès aux services de santé, l'accès à l'eau potable et la part d'enfants de moins de cinq ans victimes de la malnutrition (23). On voit par là le caractère multicritère et multiniveau de la pauvreté.

Il s'agit là, bien entendu, d'un indice moyen caractérisant un pays et non des individus. Une telle observation pose d'emblée une première interrogation. Quand on parle de pauvreté, il convient de préciser à quelle échelle : celle des pays, celle des régions (zones rurales ou urbaines notamment), celle de groupes identifiés (castes, réfugiés, femmes...) ou celle des individus. Dans son ouvrage Politique du rebelle. Traité de résistance et d'insoumission, Onfray (18) distingue trois cercles centrés sur la pauvreté : le premier cercle, celui des damnés (l'armée des mendiants dont seule l'aumône permet de maintenir un minimum vital) ; le second cercle est celui des réprouvés (malades, délinquants, clandestins, réfugiés) ; enfin, le dernier cercle est celui des exploités (emplois précaires, déplacés, prolétaires, paysans sans terre). Il est fort évident que le niveau de pauvreté, les perspectives d'évolution de cette situation, les capacités individuelles à s'en sortir varient en fonction des individus et du cercle auquel ils appartiennent.

Par rapport à l'élevage, ces considérations sont d'importance. En effet, les échelles d'analyses et les types d'individus concernés suscitent des actions différentes tant en recherche qu'au niveau du développement. S'agissant de pays pauvres, la priorité est sans doute d'ordre institutionnel afin d'accroître par des choix politiques et macro-économiques la sécurisation du secteur élevage à l'échelle nationale. Cela nécessite sans doute d'analyser les blocages de fonctionnement qui sont à l'origine d'une faible accumulation ou bien de la faible productivité des systèmes, ou les interactions complexes entre des économies nationales déséquilibrées. On peut s'interroger à ce niveau sur le rôle joué par les politiques de soutien aux exportations par les pays du Nord pour leurs produits animaux qui entrent directement en concurrence (parfois par des politiques délibérées de dumping) avec les éleveurs du Sud. Dans bien des pays tropicaux, le lait reconstitué à base de poudre importée se retrouve sur le marché national à un prix bien inférieur à celui du lait frais provenant des troupeaux laitiers locaux. De même la viande congelée issue des surplus de production de l'Union européenne a longtemps représenté un frein au développement de l'élevage des pays sahéliens principaux fournisseurs des pays côtiers consommateurs.

S'agissant de régions ou de groupes pauvres, il importera peut-être de privilégier les actions stimulant l'intégration économique de l'élevage des populations ou des régions concernées. On s'intéressera dès lors à l'existence ou non des marchés permettant l'accès aux ressources (travail, terre, éducation, crédit, information, intrants) ainsi qu'à leurs modes d'organisation (métayage, agriculture familiale, Etat-providence, projets, réseaux de solidarité...).

Pour les individus pauvres, il conviendra vraisemblablement de lever les contraintes qui limitent les capacités de capitalisation de l'individu-éleveur. Les blocages dans l'accès aux ressources peuvent être d'origine individuelle (malades, délinquants, asociaux) ou sociale (réfugiés, immigrés, paysans sans terre). Les opérations de recherche-développement ne seront donc pas de même nature si l'éleveur pauvre, pour reprendre la terminologie de Onfray (18), appartient au cercle des «damnés » (par exemple un éleveur sinistré ayant perdu tout son troupeau), à celui des « réprouvés » (un éleveur malade ou trop vieux pour assurer une saine gestion de son cheptel) ou à celui des « exploités » (comme par exemple un éleveur prolétarisé, c'est-à-dire un éleveur qui ne peut survivre qu'en louant ses services comme berger pour le compte d'un autre propriétaire). On ne peut donc analyser le rôle de l'élevage dans la lutte contre la pauvreté que si la cible est bien définie au départ et l'échelle d'analyse bien identifiée.

\section{- PAUVRES DES VILLES, PAUVRES DES CHAMPS}

Depuis la révolution industrielle, philosophes et politiques $(17,19)$ se sont surtout attardés sur la pauvreté des classes laborieuses majoritairement constituées par des populations d'origine urbaine. Les plus pauvres sont classés dans une catégorie plus citadine que rurale : le lumpenproletariat (15). Le pauvre de la tradition politique est donc d'abord un pauvre des villes. La tradition chrétienne est plus ambivalente (14). Au Brésil, la théologie de la libération qui prône une option préférentielle pour les pauvres, doit beaucoup à sa confrontation avec la misère des paysans sans terre et à l'injustice foncière qui leur est faite.

Le pauvre des champs est par ailleurs rentré en force dans l'univers médiatique grâce aux catastrophes naturelles ou non (sécheresse, sauterelles, conflits politiques...) qui ont jeté des milliers, voire des millions de paysans, d'agriculteurs, d'éleveurs dans des états d'extrême dénuement. A défaut de pouvoir se nourrir euxmêmes, les pauvres des champs ont alimenté l'émergence de l'humanitaire. Non instrumentalisé par la tradition marxiste, peu instrumentalisé par la tradition chrétienne, le pauvre des champs a surtout trouvé sa place dans une tradition humanitaire amplement relayée pour l'occasion par les institutions internationales (Banque mondiale et Fonds monétaire international) pour qui l'aide ciblée vers les pauvres représente un supplément de la stratégie d'ajustement structurel. Mais ces pauvres-là ont deux caractéristiques : (i) ils proviennent en majorité des zones rurales voire pastorales qu'ils quittent pour accroître les populations des bidonvilles des périphéries urbaines ; (ii) ils sont originaires du tiers-monde et, voulant fuir leur pauvreté, ils peuvent se porter candidat à l'émigration vers les villes du Nord. Autrement dit, ils ont tendance à s'urbaniser du fait globalement de meilleures conditions d'existence (21).

$\mathrm{Au}$ fond, la pauvreté des villes est alimentée par celle des campagnes. Et cette dualité de situation n'est pas non plus sans conséquence sur le rôle que peut jouer l'élevage dans ce contexte. L'explosion des élevages périurbains dans les villes du tiersmonde illustre, s'il en était besoin, les évolutions en cours. Si, à l'échelle mondiale, l'urbanisation conduit à une diminution de la proportion de ruraux dans la population, ceux-ci restent encore majoritaires (environ 55 p. 100 avec de fortes différences NordSud) et leur nombre absolu continue d'augmenter dans les pays du Sud. Par ailleurs, on estime globalement qu'en Afrique, 86 p. 100 de la population pauvre vit dans les zones rurales, 79 p. 100 en Asie et 66 p. 100 en Amérique latine (12).

Les causes de la pauvreté en milieu rural sont multiples et l'analyse des conditions d'existence ou d'apparition de la pauvreté relève d'approches typologiques, malheureusement peu courantes. Les conditions de milieu défavorables représentent certes un fac- 
teur important mais non essentiel : 35 p. 100 des agriculteurs pauvres dans le monde sont localisés dans des zones favorables à l'agriculture. En dehors des phénomènes transitoires et brutaux (guerres, conflits ethniques), la pauvreté est liée à l'impossibilité d'accès aux moyens de production (terre, énergie, intrants), au manque de compétences techniques (non-accès à la scolarisation) et à des situations écologiques défavorables. La pérennité du pauvre des champs dans les sociétés agraires des pays tropicaux semble le corollaire du retard maintenu de la productivité agricole dans ces pays, comparée à celle des pays industrialisés (1).

\section{QU'EST-CE QU'UN ELEVEUR PAUVRE?}

On peut simplifier en affirmant qu'il y a trois sortes d'éleveurs pauvres. Le plus démuni de tous est celui qui n'a plus d'animaux. La perte totale des animaux pour un éleveur est sans doute le summum de la déchéance, puisqu'en perdant son cheptel, l'éleveur perd son statut. On ne peut guère prétendre être éleveur quand on n'a plus rien à élever. Tout au plus, l'éleveur démuni devient-il berger pour le compte d'un autre éleveur possédant un grand troupeau, passant ainsi, comme nous l'avons évoqué plus haut, d'un statut de damné à celui d'exploité. Il existe des paysans sans terre. Pourquoi pas des éleveurs sans animaux ? On oublie cependant la perte d'identité que peut représenter pour un pasteur, la disparition de son bétail. L'appauvrissement économique se double ici d'une paupérisation culturelle et symbolique redoutable pour les sociétés, notamment pastorales, centrées sur l'animal domestique. Notons au passage que la pauvreté d'un éleveur n'est pas forcément liée à l'absence de terres disponibles. Si le paysan sans terre est pauvre parmi les pauvres, l'éleveur « hors-sol » ne fait pas partie des plus démunis.

Le second type d'éleveur pauvre est celui qui dispose d'un troupeau trop petit ou pas assez productif pour en vivre. Autrement dit la capitalisation n'est pas suffisante pour dégager des surplus en termes de produits d'origine animale commercialisables ou échangeables, voire encore plus simplement pour satisfaire les besoins alimentaires de base de l'unité familiale par autoconsommation. La notion d'effectif insuffisant a été l'objet à plusieurs reprises d'analyse détaillée de la part d'organismes de développement afin d'estimer un effectif-seuil permettant à un individu ou une famille de se situer au-delà du seuil de pauvreté. Ainsi, au Niger dans les années 70, le programme Reconstitution du cheptel définit la composition d'un troupeau minimum de redémarrage ( $\mathrm{Tmr}{ }^{1}$ ) susceptible d'assurer un revenu suffisant pour l'éleveur et sa famille ainsi qu'une capacité à rembourser l'organisme fournissant le cheptel (le remboursement pouvant se faire en espèces ou en nature, les animaux ainsi récupérés étant redistribués à d'autres éleveurs sinistrés). La composition et la taille du Tmr devaient être envisagées de telle façon que la pression de remboursement n'altérât en rien la capacité de croissance naturelle du troupeau et ce, dans l'objectif pour l'éleveur d'acquérir en quelques années un troupeau optimal requis $\left(\right.$ Tor $\left.^{2}\right)$ qui devait mettre l'éleveur sinistré à l'abri d'une nouvelle catastrophe (9).

Bien évidemment, la notion de taille insuffisante du troupeau n'a de sens qu'en intégrant l'activité d'élevage dans l'ensemble des revenus de la famille. Un petit troupeau chez un pasteur qui ne vit que de l'élevage n'est pas comparable à un petit troupeau chez un agropasteur disposant d'un important volume de récoltes. Par

\footnotetext{
${ }^{1}$ Le TMR pouvait comprendre plusieurs types : soit un troupeau mixte ( 2 vaches adultes +10 petits ruminants, ou 2 chamelles +10 chèvres), soit un troupeau homogène ( 2 vaches +3 taurillons ou 10 brebis ou 20 chèvres ou 5 vaches)

${ }^{2}$ La composition d'un Tor bovin était théoriquement la suivante : 6 vaches, 4 génisses, 4 veaux, 2 taurillons et 1 géniteur
}

ailleurs, à taille et composition égales, certains éleveurs s'en tirent mieux que d'autres grâce à des pratiques engendrant une meilleure productivité ou à des prix du marché plus attractifs.

Le troisième type d'éleveur pauvre est celui qui en dépit d'un troupeau de taille suffisante ou de bonne productivité ne parvient pas à dégager un revenu suffisant du fait d'un taux d'endettement considérable. Dans ces circonstances, l'éleveur se trouve enfermé dans une situation d'appauvrissement consécutif à des investissements disproportionnés par rapport à ses capacités de remboursement. Les crédits attribués aux éleveurs doivent tenir compte des marges disponibles en fonction de la taille et/ou de la composition du troupeau, mais aussi des spéculations attendues de l'exploitation des animaux (le lait par exemple est souvent plus rémunérateur que la viande) et des espèces concernées (la productivité numérique est d'autant plus élevée qu'il s'agit d'espèces à cycle court). Dans l'exemple cité précédemment, le Tmr devait être réfléchi dans une perspective de revenus assurant une capacité de remboursement suffisante. Notons cependant que la dette de l'éleveur peut être contractée pour d'autres raisons que des investissements en matière d'élevage, soit pour des choix économiques risqués extérieurs à l'exploitation des animaux, soit pour des raisons socioculturelles comme les frais de mariage et de dot parfois considérables dans certaines sociétés. En Inde, par exemple, la dot de mariage peut représenter une véritable catastrophe économique pour un éleveur modeste s'il n'a que des filles et pas de fils à marier.

Cette typologie a des conséquences évidentes sur les actions de recherche-développement associées. Il n'est pas envisageable en effet de mener les mêmes types d'opérations en matière de lutte contre la pauvreté selon que l'on a affaire à un éleveur sinistré qui a perdu son bétail, un petit éleveur qui n'arrive pas à capitaliser ou un éleveur surendetté. Les priorités et les modes d'intervention devront s'adapter aux situations observées. Par ailleurs, ces trois types d'éleveurs pauvres peuvent correspondre à différents stades de paupérisation d'une même personne.

\section{LA SPIRALE DE L'APPAUVRISSEMENT}

Il est généralement considéré que l'élevage des animaux domestiques dans les pays en développement demeure comme toute activité agricole une activité à risque. Un troupeau et son propriétaire peuvent être à la merci d'une sécheresse, d'une épidémie ou d'un conflit qui remet en cause la viabilité de son activité et ce, d'autant plus fortement que le cycle de reproduction des animaux élevés est long (l'élevage camélin affecté de gestation longue chez les femelles, d'une viabilité faible des produits, d'une croissance lente des jeunes et d'une puberté tardive est particulièrement risqué). Les bénéfices de l'activité d'élevage s'inscrivent généralement dans le long terme ; or, les délais de remboursement du crédit agricole sont plus souvent adaptés aux cycles des récoltes qu'à celui de la reproduction des ruminants domestiques. Si l'élevage pastoral résiste globalement mieux que l'agriculture à une sécheresse du fait de la mobilité des troupeaux (à l'inverse des terres agricoles qui par définition ne peuvent fuir les aléas climatiques), la pression pathologique peut être source d'une régulation démographique drastique, voire d'un quasi-anéantissement quand il s'agit d'épizooties très contagieuses à forte mortalité. Peste bovine et péripneumonie contagieuse bovine, en dépit des programmes internationaux de lutte, restent une menace évidente pour les populations pastorales.

Cependant, en dépit de la mobilité de son cheptel, l'éleveur subit de plein fouet en cas de crise climatique ce qu'il est convenu d'appeler la détérioration des termes de l'élevage pastoral (26). En effet, de mauvaises conditions climatiques ou une situation d'insécurité vont diminuer le disponible commercialisable en produits 
agricoles vivriers et donc conduire à leur renchérissement sur le marché. A l'inverse, ces mêmes situations de crise vont précipiter sur le marché un plus grand nombre d'animaux du fait de la politique de déstockage des propriétaires de troupeau et entraîner la chute des prix à la vente. Dans l'échange céréales-bétail en cas de crise, l'éleveur est perdant. Il devra donc surexploiter son capital animal. Si la crise dure, la mortalité naturelle augmentant, l'éleveur est condamné à emprunter auprès des commerçants pour assurer sa survie. Ainsi, dès que les conditions s'améliorent, l'éleveur se trouve endetté, avec un troupeau réduit qui rendent problématique un éventuel redressement. La situation devient dramatique quand le troupeau disparait.

Mais le cercle vicieux de l'appauvrissement fonctionne aussi chez l'agropasteur qui dispose d'une surface de terre juste suffisante ou difficile à travailler. Son activité et ses revenus peuvent être assurés s'il y a une bonne complémentarité entre l'élevage et l'agriculture notamment grâce à la culture attelée et au transfert de fertilité. En cas de crise, l'agropasteur est fragilisé par la perte éventuelle de ses animaux de trait due à la maladie, le vol ou la sécheresse. Pendant la sécheresse de 1985 en Ethiopie, l'une des causes de la famine a été la perte des nombreux bœufs de trait qui permettaient aux paysans des hauts plateaux de valoriser leurs surfaces (11). Privés de leurs animaux pour préparer la terre et labourer, les éleveurs ont dû s'endetter pour louer des paires de bœufs à des prix prohibitifs du fait de la pénurie de bêtes bien dressées. Ajouté au déficit de la production agricole lié à la baisse de pluviométrie, cet endettement a précipité les agropasteurs dans des situations d'extrême pauvreté.

D'autres exemples ont montré que certaines actions de développement peuvent précipiter une frange de la population dans la pauvreté tout en assurant un mieux-être pour une autre partie. Ainsi, une étude réalisée dans le sud de l'Inde a montré que lorsque les engrais chimiques remplacent le fumier de vache, ce sont les hommes plutôt que les femmes qui désormais épandent l'engrais parce que les femmes n'ont pas accès à l'information diffusée par les services de vulgarisation. Cela conduit à des pertes d'emploi pour les femmes et à une diminution des revenus du ménage (21).

Il existe sans doute bien d'autres scénarios de paupérisation. Leur connaissance n'est pas sans intérêt, car ils donnent des pistes pour casser le mouvement vers un état de pauvreté croissant, ce dernier étant perceptible à deux niveaux : approfondissement des détresses individuelles et augmentation du nombre de personnes touchées. L'ensemble de ces considérations doit permettre maintenant de situer le rôle de l'élevage dans la lutte contre la pauvreté.

\section{ם ElEVAGE et lutte CONTRE LA PAUVRETE}

Les pauvres des pays en développement tirent généralement leurs moyens de subsistance de leur travail, notamment de celui provenant de la valorisation de leurs terres ou des terres communautaires. L'élevage, de ce point de vue, joue un rôle multiple dans la lutte contre la pauvreté. Des études de cas dans différents pays du Sud ont montré notamment que dans une même communauté, la part de l'élevage dans les revenus est en moyenne plus élevée dans les ménages pauvres comparés aux ménages les plus nantis (tableau I).

L'élevage intervient à cinq niveaux principaux pour répondre à l'allègement de la pauvreté. Ces niveaux ne sont évidemment pas exclusifs.

Le premier niveau d'intervention de l'activité d'élevage se caractérise par son rôle de sécurisation. Le pauvre se définit d'abord par sa vulnérabilité : vulnérabilité sociale (risque d'exclusion), économique (perte totale de revenus), voire physique (risque d'incapacité physique). Par ses produits, ses revenus et la place qu'il confère dans la société, l'élevage représente un élément fort de sécurisation. Sur le plan alimentaire d'abord (sécurisation alimentaire), la part importante d'autoconsommation permet aux membres de la famille de l'éleveur d'avoir accès à des protéines animales (lait, œufs, viande) qui peuvent être parfois considérées comme des produits de luxe : c'est le cas par exemple du lait qui est souvent disponible localement au prix du marché mondial, donc très cher pour des revenus modestes. A noter que cette sécurité alimentaire ne concerne pas uniquement les éleveurs au sens strict du terme. Des projets humanitaires ont suggéré la mise en place de petits élevages (volailles, lapins) dans les camps de réfugiés pour assurer un minimum d'apport en protéines animales à des populations démunies. De tels projets ciblent en priorité les groupes les plus vulnérables dans les ensembles familiaux (enfants, femmes allaitantes ou enceintes). L'élevage urbain joue également ce rôle auprès de populations issues de l'exode rural.

L'élevage prend aussi sa part dans la sécurisation de l'outil de travail et il peut être utile de rappeler ici l'importance de la culture attelée dans la mise en valeur agricole et, plus globalement, le rôle de la force animale dans les activités de transport et de travail agricole. Il a été souligné plus haut combien la perte des animaux de labour était préjudiciable à la survie des exploitations paysannes. La présence de bœufs de trait représente non seulement une garantie pour les travaux agricoles, mais aussi une source potentielle de revenu par la location auprès d'autres paysans.

L'activité d'élevage est également un atout dans le maintien de la fertilité des sols par le transfert des matières organiques animales vers les terres de culture. Il s'agit d'une assurance de productivité accrue du travail de la terre propice à l'amélioration du revenu du paysan.

Plus généralement, l'activité d'élevage remplit une fonction d'épargne à court terme (ce rôle est joué de façon prépondérante par les espèces à cycles courts) qui sécurise le producteur et sa famille dès lors qu'elle doit faire face à des dépenses prévues (frais de scolarité, impôts et taxes diverses) ou non (accident, maladie, frais de funérailles) nécessitant la mobilisation rapide de liquidités. La vente d'une part du bétail dans un tel contexte permet à l'éleveur de satisfaire à une demande rapide de fonds tout en évitant de s'enfoncer dans une logique d'endettement dont on a vu le risque d'appauvrissement que cela peut déclencher.

Il est intéressant de noter à ce stade, les mécanismes de sécurisation mis en place par les acteurs économiques vivant exclusivement de l'élevage pastoral. Les mécanismes de transmission et de circulation du bétail remplissent par exemple une fonction assurant un certain égalitarisme des groupes domestiques constituant la communauté pastorale. La redistribution des produits (confiage, prêts) ou de bétail contre travail (contrats de gardiennage) contribue à maintenir une certaine cohésion sociale (3).

\section{Tableau I}

Part du revenu issue de l'élevage chez les ménages riches et les familles pauvres ( $d$ 'après Delgado et coll., 1999, Ifpri, adapté par I'Ilri, 1999)

\begin{tabular}{lrc} 
Pays (zone) & Le plus riche $(\%)$ & Le plus pauvre (\%) \\
\hline Sénégal (zone aride) & 14 & 24 \\
Sénégal (semi-aride) & 8 & 10 \\
Philippines & 10 & 23 \\
Pakistan & 9 & 25 \\
Egypte & 14 & 63
\end{tabular}


La sécurisation assurée par le bétail est même, plus globalement, d'ordre monétaire : au Brésil, pendant la période d'hyperinflation du début des années 90 , le bétail représentait un refuge contre l'érosion monétaire. Plus généralement, le bétail est le seul moyen de placer un surplus de revenu en l'absence de banque accessible. Enfin, certains produits comme le lait, de par leur disponibilité quotidienne pour la mise sur le marché, permettent un accès régulier à une trésorerie, ce qui n'est pas possible avec la plupart des produits végétaux dépendants d'une récolte en général annuelle.

Le second rôle de l'élevage est celui qui permet au propriétaire d'assurer une capitalisation, meilleur moyen de sortir de la spirale de l'appauvrissement. Ce n'est sans doute pas par hasard que les termes cheptel et capital ont la même étymologie. On peut émettre l'hypothèse que le bétail a représenté au cours de l'histoire la première forme de capitalisation. La littérature surabonde de critiques sur la pratique de l'accumulation de bétail notamment chez les pasteurs aux dépens de la productivité. Il ne faut pourtant voir là en priorité qu'une stratégie limitant la fragilisation du statut social et économique de l'éleveur en cas de perte massive lors de crises climatiques ou de conflits. Cette capitalisation procède souvent par étapes. Dans les pays du Sahel, il a bien été montré que les pasteurs victimes d'une sécheresse reconstituaient leurs troupeaux de préférence par l'acquisition de petits ruminants, moins coûteux et plus productifs. A un stade ultérieur, la capitalisation s'appuie sur le cheptel bovin et éventuellement camélin. Mais l'élevage peut aussi être un tremplin pour une autre activité, ce qui semble être le cas pour certains paysans des fronts pionniers amazoniens : l'activité d'élevage permet de se constituer un capital assez rapidement susceptible d'être réinvesti dans d'autres activités agricoles, artisanales ou semi-industrielles. Il y a là toute une trajectoire qui sur le plan économique est riche d'enseignement.

Le troisième rôle de l'élevage dans le cadre de la lutte contre la pauvreté est représenté par sa fonction de diversification des activités et des revenus. Il ne s'agit pas, d'ailleurs, des seules activités agricoles. En milieu urbain, l'élevage est une source complémentaire de revenus pour des populations exerçant d'autres métiers que celui de paysan : commerçants, artisans ou fonctionnaires. Chez ces derniers, dans les contextes économiques récents de certains pays d'Afrique caractérisés par de considérables retards de salaire, l'élevage urbain, même à petite échelle a permis une diversification des sources de revenus. L'avantage de la diversification est qu'elle diminue les risques inhérents à une production unique. Par ailleurs, de par les variations saisonnières du marché, les opérateurs économiques peuvent jouer sur plusieurs produits dont les fluctuations ne suivent pas les mêmes lois, s'assurant ainsi un revenu régulier : ainsi le lait est surtout disponible en saison des pluies lorsque les pâturages sont verts, alors que les céréales ne sont récoltées qu'en début de saison sèche. De nombreuses études socio-économiques menées en Afrique noire ont bien mis en évidence que les ménages ayant choisi la diversification des activités étaient globalement moins vulnérables que ceux caractérisés par une monoactivité (24). Par ailleurs, l'activité d'élevage par son découplage par rapport au cycle saisonnier des récoltes peut jouer un rôle tampon souvent bienvenu. La diversification est aussi une façon d'utiliser une main-d'œuvre familiale autrement peu, voire non-productive. On peut également citer pour mémoire, le rôle d'activités connexes centrées sur l'animal telles que la chasse ou l'élevage d'espèces non-conventionnelles, comme il l'a bien été montré dans l'ouvrage collectif Faune sauvage. La ressource oubliée (4). Ces activités s'inscrivent totalement dans les logiques de diversification.

L'activité qui consiste à élever des animaux représente également un puissant facteur d'intégration économique dès lors que cela dépasse le stade de l'autoconsommation. La possession d'animaux de rente permet de passer d'une situation d'assisté à celle d'acteur économique. Par ses produits, l'éleveur intègre des logiques de filières, s'inscrit dans des stratégies d'optimisation des crédits et des revenus. Les programmes de crédit visant à répondre aux besoins des pauvres (par exemple la Grameen Bank au Bangladesh) accordent assez facilement des prêts pour des petits projets d'élevage dont les femmes sont très demandeuses : 45 p. 100 des femmes emprunteuses choisissent un projet d'élevage (contre 19 p. 100 pour les hommes). En moyenne, le tiers des emprunts est destiné à l'achat de bétail ou à des projets concernant les volailles ou la pêche. L'éleveur peut aussi être associé, moyennant un investissement minimum, à la transformation de ses produits (produits laitiers notamment) qui assure une plus-value rémunératrice. Mais plus globalement, le développement rural est d'autant plus efficace qu'une fiscalité équitable permet aux plus pauvres de valoriser leurs investissements, ce qui n'est pas le cas par exemple dans de nombreux pays d'Amérique latine.

Enfin, l'élevage demeure un important facteur d'intégration sociale. On sait le prestige associé à un grand troupeau dans bien des sociétés pastorales où les transactions de bétail au moment des événements importants de l'existence (dot pour le mariage, préhéritage, sacrifices au moment du décès) sont primordiales. Le bétail assure un statut social aux individus, une reconnaissance qui signifie la sortie de l'état de pauvreté. L'habitude de perpétuer une activité d'élevage en situation urbaine n'est pas étrangère non plus à ce souci de reconnaissance sociale qui dans l'imaginaire collectif de bien des pays tropicaux est mieux assurée par le bétail que par des cultures maraîchères par exemple. Les pasteurs ont développé depuis longtemps des stratégies de survie par un partage du risque dans l'espace (mobilité des troupeaux), entre espèces (élevage de troupeaux plurispécifiques) et dans le temps (confiage et retour de confiage). Ce dernier point permet de construire un réseau d'alliances et de solidarité sociale reposant entièrement sur le cheptel (le habbanae des Peuls ou l'intila des Afar par exemple). L'appartenance à un tel réseau est la marque d'une intégration sociale poussée (10), indispensable aux individus isolés pour résister à la spirale de l'appauvrissement.

Dans les pays musulmans, il existe un système traditionnel de redistribution des richesses particulièrement efficace quand il s'agit d'élevage. Par exemple, en Mauritanie, on distingue trois types d'entraides : la première, moralement obligatoire, est la zeqat et les deux autres, non obligatoires mais moralement valorisantes pour l'accès au paradis, sont appelées hobs et lmiha. La zeqat consiste à donner à un pauvre un chamelon de 3 ans pour toute unité de 50 têtes, ou un veau de 2 ans pour toute unité de 30 vaches, ou un chevreau/agneau de 1 an pour toute unité de 100 brebis/chèvres. Un calcul rapide indique que pour un pays comme la Mauritanie cela permet la redistribution d'un capital de 230 millions de dollars aux familles démunies. Certes, la zeqat est également valable sous forme de dons de céréales, d'or ou d'argent. Mais le bétail, par le capital productif qu'il représente, constitue un réel appoint dans la lutte contre la pauvreté. Le hobs consiste à fournir un lot d'animaux afin que le pauvre puisse bénéficier des produits et se constituer ainsi à peu de frais un troupeau. L'Imiha est un peu équivalent mais le don est représenté uniquement par le lait produit par les animaux attribués.

\section{- QUELLES QUESTIONS POUR LA RECHERCHE?}

Il est illusoire de considérer que la lutte contre la pauvreté est d'abord une question technique. Illusoire et pernicieux, car cela dédouane complètement les décideurs politiques et économiques. La pauvreté n'est pas un fait technique. C'est un fait économique et 
politique. La pauvreté est d'abord le résultat de choix opérés à des échelles nationale et/ou internationale, sur lesquels la communauté scientifique n'a de prise qu'en tant que communauté de citoyens.

Néanmoins, la recherche peut apporter des réponses techniques permettant aux pauvres de sortir individuellement de la spirale de la pauvreté dès lors que des questions techniques peuvent être identifiées. Par ailleurs, l'analyse de la situation de pauvreté chez un éleveur implique un positionnement clair de la recherche sur l'objet d'étude. S'il s'agit de lutter contre la pauvreté, alors il convient en effet de travailler sur des concepts (élevage familial par exemple), des animaux (races locales), des ressources (parcours naturels, fourrages locaux) ou des productions (produits traditionnels) susceptibles de répondre aux besoins et interrogations des plus pauvres. Il s'agit aussi de fournir aux décideurs des indicateurs fiables permettant d'évaluer l'état de pauvreté ou, d'une manière plus dynamique, l'évolution de la situation économique d'individus ou de groupes (indicateurs d'appauvrissement ou, à l'inverse d'enrichissement) ou bien encore d'indicateurs de la cohésion sociale illustrés par exemple par l'écart entre riches et pauvres.

Concernant l'activité d'élevage, nous avons vu que son premier rôle consistait à sécuriser les populations d'agriculteurs. Tout moyen susceptible de contribuer à cette sécurisation milite dans le sens d'une amélioration des revenus de l'éleveur. Une meilleure protection sanitaire, une amélioration de la productivité, une évaluation des impacts sur l'environnement sont autant de réponses qui relèvent de la recherche en élevage. De même, l'analyse des stratégies de capitalisation et de diversification peut contribuer à une meilleure compréhension des mécanismes possibles de dépaupérisation et des dynamiques à encourager. Les études des filières et de la place de l'élevage dans la société participent à l'identification des contraintes techniques et socioculturelles qui entravent le développement de l'élevage et de la transformation de ses produits.

Cependant, si l'approche technique est indispensable pour aborder certains aspects de la lutte contre la pauvreté (déterminants techniques de la productivité, modes de production alternatifs), elle se doit d'être complétée par des approches socio-économiques qui permettent notamment une analyse pertinente des formes de pauvreté rurale (systèmes de production risqués, faible accès aux ressources) ou urbaine (déterminants économiques de l'exode rural, système économique informel) et surtout par des approches en sciences humaines (déterminants historiques, géographiques, sociaux ou politiques des situations de pauvreté). La question de la dynamique de la pauvreté est en effet une question complexe qu'il convient d'aborder en collaboration au sein de démarches pluridisciplinaires.

S'il est utile de rappeler ici que les productions animales jouent un rôle essentiel dans la dynamique de la pauvreté, notamment pour proposer des solutions à la sortie du cercle vicieux de l'appauvrissement ou pour accéder aux populations marginales qui pratiquent souvent l'élevage, il ne faut pas oublier que les déterminants techniques ne sont que les résultats de modes d'organisations productifs et sociaux dans lesquels les productions animales sont mises en œuvre.

Quelques pistes de recherches en productions animales peuvent être ainsi proposées notamment pour permettre :

- l'identification des populations pauvres de la planète et de leurs modes de fonctionnement (populations pastorales, petit élevage, élevage laitier en milieu rural et périurbain, agro-éleveurs...) ;

- l'identification des mécanismes d'intégration entre l'agriculture et l'élevage en zone rurale, et des mécanismes moteurs du développement ;

- l'étude de l'impact du développement économique périurbain sur la pauvreté des différentes populations cibles (compétitivité des différents systèmes et voies d'amélioration de cette compétitivité).
QUELLES CONSEQUENCES POUR LE DEVELOPPEMENT?

Pendant plusieurs décennies, chacun pouvait considérer que la pauvreté était soluble dans le développement. Dans les faits, les relations entre développement et pauvreté se sont révélées plus complexes que ne le suggéraient les représentations précédentes : la « modernisation » des sociétés n'a pas provoqué la disparition automatique de la pauvreté, bien au contraire. Par ailleurs, les organismes de développement fonctionnent beaucoup en comptant sur l'imitation des individus les plus aptes ou les plus réceptifs à l'innovation (cf. le concept de « la ferme-modèle » ou de « l'éleveur-pilote »). Un tel processus a tendance à écarter les moins accessibles et donc à creuser davantage l'écart entre les forces vives et les laissés-pour-compte.

La réflexion qui s'ensuit pour le développement de l'élevage pourrait s'inspirer de celle engagée sur les enjeux scientifiques. Un projet de développement de l'élevage orienté vers la lutte contre la pauvreté devrait notamment pouvoir mettre en œuvre les mécanismes permettant la levée des contraintes identifiées par la recherche. L'organisation des programmes de prévention sanitaire, la formation des éleveurs aux techniques de soins primaires, la levée des obstacles socioculturels à l'amélioration des pratiques d'élevage, la démarche participative à la prise en compte des facteurs environnementaux, le soutien à l'émergence d'organisations paysannes susceptibles de faciliter les flux d'intrants et de services, sont autant d'engagements qui s'inscrivent dans les objectifs des organismes de développement ciblés sur l'activité d'élevage en particulier ou agricole en général. Ces orientations, qui ne sont pas nouvelles, n'éliminent pas la nécessité d'appuyer des politiques macroéconomiques œuvrant dans le même sens (politique fiscale favorisant la redistribution, protection des marchés des produits). A ce titre, le rapport de Delgado et coll. (7) consacré à la nouvelle révolution alimentaire identifie globalement quatre piliers sur lesquels les politiques de développement doivent s'appuyer pour permettre de répondre aux besoins des plus démunis : (i) l'intégration verticale grâce à des institutions collectives ; (ii) l'élimination des distorsions de politiques faisant pencher la balance du côté des grands producteurs (latifundia, fermes d'Etat) aux dépens de l'agriculture familiale et paysanne, en particulier par l'amélioration du système de crédit pour les petits producteurs ; (iii) la création de biens et services publics, en particulier les infrastructures de transport et de communication ; (iv) la promotion d'institutions régulatrices pour affronter les problèmes d'environnement et de santé publique. Tant que les politiques mises en ouvre ne permettent pas d'ouvrir une perspective économique aux pauvres, les actions de développement risquent de n'avoir d'efficacité qu'à la marge. En tout état de cause, le développement de l'élevage doit pouvoir s'appuyer sur une articulation entre l'amélioration des techniques d'élevage et la mise en œuvre de politiques plurisectorielles.

\section{CONCLUSION}

Ces réflexions très générales n'ont cependant pas abordé les questionnements de fond suivants :

1. Si les programmes d'urgence visent à n'en point douter les plus démunis (réfugiés, déplacés, victimes des sécheresses, d'épidémies et autres calamités), les programmes de développement touchent plus difficilement ceux qui en ont le plus besoin. Les plus pauvres s'inscrivent difficilement dans une stratégie de développement. Tout au plus, recherchent-ils une sécurisation minimale assurant leurs besoins physiologiques de base. On atteint rarement les plus démunis. A moins que l'objectif ne soit précisément d'avoir des 
informations sur la frange la plus extrême des catégories pauvres, l'analyse des études et des projets d'élevage (et d'autres activités sans doute) montre clairement que les éleveurs pris en compte dans les réseaux de suivi, les enquêtes sanitaires ou zootechniques, les expérimentations en milieu paysan, etc., ne sont jamais les plus marginalisés. Combien d'études s'appuient sur les choix dictés par les chefs traditionnels ou les potentats locaux, partenaires « politiquement » incontournables ? Au Burundi, par exemple, un projet d'amélioration génétique du bétail local par insémination artificielle s'est appuyé sur l'élaboration d'un cahier des charges (état corporel de la mère, hygiène du bâtiment, qualité des ressources fourragères...) qui, de fait, écartait les plus pauvres des producteurs. Plus généralement, " aucune enquête statistique ne parvient à saisir les populations situées aux extrêmes de la hiérarchie sociale » (2), les plus pauvres et les plus riches. Quand un pauvre ne s'exprime pas, qui va entendre sa parole ?

2 . Le « choix des pauvres » ne répond pas aux canons habituels du discours sur le développement qui par essence s'appuie préférentiellement sur les éléments les plus dynamiques des catégories socioprofessionnelles visées. Ce qui fait dire, par exemple, à des responsables paysans du Sud, qu'il vaut mieux soutenir « ceux qui travaillent, plutôt que les plus pauvres » (8). Au-delà des considérations éthiques qu'implique l'engagement auprès des producteurs les plus démunis, il faut effectivement s'interroger sur les voies les plus appropriées pour le développement du plus grand nombre. Un soutien accru aux plus pauvres est-il le chemin le plus approprié pour accroître l'efficacité économique de la masse des éleveurs ?

3. La pauvreté n'est pas nouvelle. Si elle augmente à l'échelle de la planète, c'est plus en effectif qu'en proportion, bien que dans le même temps, on peut constater une croissance incontestable des inégalités (les 20 p. 100 les plus riches ont des revenus 82 fois plus élevés que les 20 p. 100 les plus pauvres) (20). Aussi, on peut s'étonner de l'engouement soudain pour un thème récurrent dans les pays tropicaux, mais aussi dans les pays industrialisés. L'effet mode nécessite un affichage plus conséquent sur le thème de la lutte contre la pauvreté. Mais ce choix, certes fort respectable au regard des engagements de chacun, ne masque-t'il pas des faillites politiques et macroéconomiques ? Le thème de la lutte contre la pauvreté n'est-il pas l'arbre qui cache la forêt d'une mondialisation qui fabrique des exclus (20)?

Si l'élevage est de fait un moyen pour les hommes de sortir de la pauvreté, il n'est qu'une solution marginale à un problème qui dépasse les faits techniques sur lesquels nous sommes susceptibles d'agir.

\section{Remerciements}

Je tiens à remercier G. Duteurtre, J.P. Boutonnet, H. Guerin, G. Tacher et B. Toutain pour leurs remarques qui m'ont permis d'améliorer le texte initial.

\section{BIBLIOGRAPHIE}

1. BAIROCH P., 1992. Le tiers-monde dans I'impasse. Paris, France, Gallimard, 660 p. (Coll. Folio)

2. BIHR A., PFEFFERKORN R., 1999. Les riches, terra incognita des statistiques. Le monde diplomatique, mai 1999, p. 15

3. BONTE P., 1978. Egalité et inégalité chez les pasteurs nomades. In : Production pastorale et société. Supplément à MSH-Informations. Paris, France, Maison des sciences de l'homme, $46 \mathrm{p}$.

4. CHARDONNET P. éd., 1995. Faune sauvage africaine. La ressource oubliée. Tomes I et II. Bruxelles, Belgique, Ceca-CE-Ceea, 704 p.

5. CORTEN A., 1998. Le discours de la pauvreté de la Banque mondiale. Lang. Soc., $n^{\circ} 85,5-24$.

6. Courrier de la planète, 1997. Vieille charité et nouvelle pauvreté. Montpellier, France, Solagral. ( $\left.n^{\circ} 38\right)$

7. DELGADO C., ROSEGRANT M., STEINFELD H., EHUI S., COURBOIS C., 1999. Livestock to 2020. The next food revolution. Washington, DC, USA, IFPRI, $17 \mathrm{p}$.

8. DIALLO P.M., VOGEL J., 1996. L'Afrique qui réussit. Vie et combats d'un leader paysan guinéen. Paris, France, Syros. (Coll. Ateliers du développement)

9. FAYE B., 1979. Gestion théorique des troupeaux de reconstitution. Programme Reconstitution du cheptel. Niamey, Niger, PRC-CM, 83 p.

10. GALLAIS J., 1989. Une géographie politique de I'Ethiopie. Le poids de I'Etat. Paris, France, Economica, 213 p.

11. GREESELS G., JUTZI S., 1986. Relance de I'agriculture après la sécheresse. Le projet bœuf/semences du Cipea. Cipea Actual., 5 : 1-3.

12. HERVIEU B., 1996. Du droit des peuples à se nourrir eux-mêmes. Paris, France, Flammarion, $133 \mathrm{p}$.

13. ILRI, 1999. Rapport annuel. Mettre la révolution dans le secteur de l'élevage au service des pauvres. Nairobi, Kenya, Ilri, 77 p.
14. JACQUARD A., 1996. Le souci des pauvres. L'héritage de François d'Assise. Paris, France, Calmann-Levy, $134 \mathrm{p}$.

15. LABICA G., BENSUSSAN G., 1985. Dictionnaire critique du marxisme, $2^{\mathrm{e}}$ édn. Paris, France, PUF, 1240 p.

16. LEWIS O., 1963. La famille Sanchez. Paris, France, Gallimard, 638 p.

17. MARX K., 1964. Misère de la philosophie. Réponse à la philosophie de la misère de $M$. Proudhon, 1847. Paris, France, Union générale d'éditions. (Coll. 10-18)

18. ONFRAY M., 1997. Politique du rebelle. Traité de résistance et d'insoumission. Paris, France, Grasset.

19. PROUDHON P.J., 1964. Système des contradictions économiques ou philosophie de la misère, 1846. Paris, France, Union générale d'éditions. (Coll. 10-18)

20. RAMONET I., 1997. Géopolitique du chaos. Paris, France, Galilée.

21. Rapport sur le développement dans le monde, 1990. La pauvreté. Washington, DC, Etats-Unis, Banque mondiale, 287 p.

22. Rapport mondial sur le développement humain, 1992. La répartition des revenus dans le monde. Rapport Pnud. Paris, France, Economica, 229 p.

23. Rapport mondial sur le développement humain, 1997. L'indice de pauvreté humain. Rapport Pnud. Paris, France, Economica, 270 p.

24. REARDON T., 1994. La diversification des revenus au Sahel et ses liens éventuels avec la gestion des ressources naturelles par les agriculteurs. In : Benoit-Cattin M., De Grandi J.C., éds, Promotion de systèmes agricoles durables dans les pays d'Afrique soudano-sahélienne. Rome, Italie, Fao-Cta-Cirad, p. 205-217.

25. RUFIN J.C., 1991. L'empire et les nouveaux barbares. Paris, France, Lattès, $247 \mathrm{p}$.

26. THEBAUD B., 1988. Elevage et développement au Niger. Genève, Confédération helvétique, Bureau international du travail, 82 p.

Reçu le 08.11.2001, accepté le 06.09.2002 


\section{Summary}

Faye B. Role of Animal Husbandry to Alleviate Poverty

Poverty reduction is one of the top priorities of many international authorities. In this context the role played by animal husbandry deserves to be specified. Poverty is defined by economic, but also by social and cultural parameters. It is expressed at different levels such as the country, region, social category, and individual levels. Urban and rural poverty are closely related. Three types of poor farmers might be distinguished: farmers who lost their herds, farmers whose herds are too small or little productive, indebted farmers. These three types might correspond to various stages of increasing poverty. In response to the challenge of poverty reduction animal husbandry intervenes at the following five levels: security, capitalization, diversification, economical integration, and social integration. The place held by animal husbandry brings to light the need for some research and development issues to be specified.

Key words: Animal husbandry - Poverty - Economics - Animal production - Productivity - Food security.

\section{Resumen}

Faye B. Papel de la producción animal en la lucha contra la pobreza

La lucha contra la pobreza forma parte de las prioridades de numerosas organizaciones internacionales y el lugar de la producción animal dentro de esta perspectiva amerita mayor precisión. La pobreza surge de criterios económicos, pero también sociales y culturales, manifestándose a varios niveles (país, regiones, categorías sociales, individuos). La pobreza urbana y la pobreza rural se encuentran estrechamente ligadas. Podemos clasificar a los productores en tres categorías: aquellos que perdieron su hato, los que sólo disponen de un hato muy pequeño o con una producción demasiado baja para sobrevivir, los productores endeudados. Estas tres categorías pueden corresponder a diferentes fases de un empobrecimiento creciente. Con el fin de responder al desafío de la lucha contra la pobreza, la producción animal interviene a cinco niveles: seguridad, capitalización, diversificación, integración económica, integración social. Esta posición de la producción animal nos lleva a cuestionamientos de investigación y de desarrollo que ameritan ser precisadas.

Palabras clave: Ganadería - Pobreza - Economía - Producción animal - Productividad - Seguridad alimentaria. 